\title{
PROGRESIÓN DE LA RESPUESTA INFLAMATORIA SISTÉMICA EN PACIENTES CON APENDICITIS*
}

\author{
Drs. Marcelo Beltrán S. ${ }^{1,2,3}$, Rodrigo Barrera C. ${ }^{1,2,3}$, Ramón Díaz J. ${ }^{1,2}$, \\ Luis Jaramillo R. ${ }^{1,2,3}$, Cristóbal Larraín T. ${ }^{1,2,3}$, Catalina Valenzuela V. ${ }^{1,2}$ \\ 1 Servicio de Cirugía Hospital San Juan de Dios de La Serena. \\ 2 Unidad de Emergencias Hospital San Juan de Dios de la Serena. \\ 3 Departamento de Clínicas, Facultad de Medicina, Universidad Católica del Norte, Coquimbo. \\ Chile.
}

\begin{abstract}
\section{Progression of the systemic inflammatory response in patients with appendicitis}

Introduction: The systemic inflammatory response has been described in patients with appendicitis. However, its progression from the onset of symptoms to diagnosis has not been characterized. The purpose of this study was to describe and characterize the systemic inflammatory response to appendicitis. Patients and Methods: A descriptive study was conducted. One-hundred eighty-three patients were studied divided into four groups from onset of symptoms to diagnosis. Primary outcome measure was to determine the systemic inflammatory response to appendicitis according to the established groups of time intervals. Secondary outcome measure was the analysis of C-reactive protein for the same purpose. Results: The systemic inflammatory response variables according to diagnostic intervals showed non-significant differences on white blood cells count. Temperature and heart rate rises constantly after $48 \mathrm{~h}$ reaching their peak after $72 \mathrm{~h}(\mathrm{p}=0.001)$; the respiratory rate rises after $73 \mathrm{~h}(\mathrm{p}<0.0001)$. After $73 \mathrm{~h}$ most patients had 3 or 4 systemic inflammatory response criteria $(p<0.0001)$. C-reactive protein levels rise progressively showing higher levels after 48 hours $(p$ $=0.005)$. Conclusions: The inflammatory response to appendicitis is progressive, being more marked along the timeline from onset of symptoms to diagnosis.
\end{abstract}

Key words: SIRS, appendicitis, C-reactive protein, inflammatory response.

\section{Resumen}

Introducción: La progresión de la respuesta inflamatoria sistémica en pacientes con apendicitis desde el inicio de los síntomas hasta el diagnóstico no ha sido caracterizada. Los objetivos del presente estudio son describir y caracterizar la respuesta inflamatoria sistémica a la apendicitis. Pacientes y Métodos: Se realizó un estudio descriptivo. Ciento ochenta y tres pacientes fueron estudiados y divididos en cuatro grupos de acuerdo al tiempo transcurrido entre el inicio de los síntomas y el diagnóstico. El objetivo principal fue de-

\footnotetext{
*Recibido el 9 de enero de 2014 y aceptado para publicación el 2 de febrero de 2014.

Los autores no refieren conflictos de interés.

Correspondencia: Dr. Marcelo A. Beltrán S. beltran_01@yahoo.com - ClinicalTrials.gov Identifier: NCT01718171
} 
terminar la respuesta inflamatoria sistémica a la apendicitis de acuerdo a los grupos de tiempo establecidos. El objetivo secundario fue el análisis de la proteína $C$ reactiva para el mismo propósito. Resultados: La variable leucocitosis de la respuesta inflamatoria sistémica de acuerdo con los períodos de evolución no mostró diferencias significativas. La temperatura y la frecuencia cardíaca se elevan constantemente después de $48 \mathrm{~h}$ llegando a su nivel máximo después de las $72 \mathrm{~h}(\mathrm{p}=0,001)$. La frecuencia respiratoria aumenta después de 73 $\mathrm{h}$ de evolución $(\mathrm{p}<0,0001)$. Después de las $73 \mathrm{~h}$ desde el inicio de los síntomas, la mayoría de los pacientes presentan 3 ó 4 variables del síndrome de respuesta inflamatoria sistémica. La proteína $C$ reactiva se eleva progresivamente mostrando sus valores máximos después de $48 \mathrm{~h}(\mathrm{p}=0,005)$. Conclusiones: La respuesta inflamatoria sistémica a la apendicitis es progresiva y más marcada a lo largo del tiempo desde el inicio de los síntomas hasta el diagnóstico.

Palabras clave: SIRS, appendicitis, proteína $\mathrm{C}$ reactiva, respuesta inflamatoria.

\section{Introducción}

La respuesta inflamatoria a patología quirúrgica severa ha sido objeto de estudio intensivo ${ }^{1-8}$. La respuesta regional y sistémica ha sido descrita ${ }^{2,4}$, los parámetros inflamatorios han sido aplicados a algoritmos diagnósticos ${ }^{3-6} ; \mathrm{y}$ puntuaciones diagnósticas incorporando parámetros inflamatorios han sido desarrolladas ${ }^{7-10}$. El síndrome de respuesta inflamatoria sistémica (SIRS) refleja la respuesta del organismo humano a la injuria ${ }^{11}$. La respuesta sistémica continua en pacientes con procesos inflamatorios e infecciosos puede resultar en disfunción y falla orgánica múltiple ${ }^{1,2,5}$. Aun cuando la presencia del SIRS ha sido descrita en pacientes con apendicitis, su evolución progresiva asociada a sintomatología clínica no ha sido descrita o caracterizada. Así como en otras patologías, la respuesta inflamatoria del paciente a la apendicitis progresa paulatinamente. El objetivo del presente estudio fue describir la respuesta inflamatoria sistémica a la apendicitis desde el inicio de los síntomas hasta el diagnóstico en pacientes operados de urgencia por apendicitis.

\section{Pacientes y Métodos}

\section{Diseño}

En nuestra institución se operan cada año un promedio de 250 pacientes adultos por apendicitis ${ }^{12}$. Por lo tanto, para realizar este estudio se requirió una muestra de 152 pacientes, la cual tendría un poder de $80 \%$, error de $5 \%$ y $95 \%$ intervalos de confianza (IC95\%). Durante un año se incluyeron prospectivamente en el estudio 268 pacientes, de ellos 85 casos que no cumplieron con los criterios de inclusión fueron excluidos. En total 183 pacientes fueron incluidos de acuerdo con los siguientes criterios: mayores de 15 años de edad, operados de urgencia por apendicitis, con diagnóstico confirmado por histopatología. Los criterios de exclusión fueron: pacientes con condiciones patológicas inmunodepresoras o inmunosupresoras, biopsia que describía apéndice normal y pacientes sometidos a apendicectomía como parte de otro procedimiento electivo. Los pacientes fueron divididos en cuatro grupos de acuerdo al intervalo de tiempo entre el inicio de los síntomas y el diagnóstico: Grupo 1 de 0 a 24 h, Grupo 2 entre 25 y 48 h, Grupo 3 de 49 a 72 h y Grupo 4 más de $73 \mathrm{~h}$. Al ingreso se consignaron los datos clínicos, el tiempo de evolución desde el inicio de los síntomas hasta el diagnóstico, el recuento de leucocitos (RL), el valor de la proteína $\mathrm{C}$ reactiva (PCR) y la puntuación SIRS. El objetivo principal fue determinar la respuesta inflamatoria sistémica esperada a la apendicitis de acuerdo a los grupos de tiempo establecidos utilizando los criterios SIRS. El objetivo secundario es el análisis de la PCR como parámetro inflamatorio habitualmente incluido dentro del estudio de los pacientes con sospecha de apendicitis.

\section{Relevancia clínica del SIRS}

La relevancia clínica del SIRS en paciente con apendicitis se estudió correlacionando las puntuaciones SIRS con apendicitis perforada y las complicaciones postoperatorias.

\section{Definiciones}

El intervalo de tiempo entre el inicio de los síntomas y el diagnóstico se designó como "intervalo diagnóstico". Se utilizaron las definiciones establecidas para los criterios del SIRS ${ }^{5,11}$. Se asignó un punto por cada criterio positivo lo que da como resultado una puntuación de 0 a 4 puntos ${ }^{5,11}$. Se consideró que un paciente tenía SIRS cuando presentó 2 o más puntos ${ }^{11}$. Los valores normales de nuestro laboratorio institucional para PCR son de 0 a $5 \mathrm{mg} / \mathrm{L}$ y para $\mathrm{RL}<11.300 \mathrm{~mm}^{3}$.

\section{Estadística}

Las variables continuas se reportaron como promedio \pm desviación estándar y se analizaron con el test de análisis de varianza. Las variables categóricas se reportaron como frecuencias y se analizaron con el $\chi^{2}$. Las comparaciones se realizaron con el test 
Wilcoxon-Bonferroni. Las correlaciones entre la puntuación SIRS, apendicitis perforada y complicaciones postoperatorias fueron evaluadas con el test de Pearson. La base de datos se trabajó con el software SPSS 11,0.

\section{Resultados}

\section{Características generales}

La serie se compuso de 107 hombres $(58,5 \%)$ y 76 mujeres $(41,5 \%)$, la edad promedio fue $29,7 \pm$ 12 años (15-77). En 45 pacientes (25\%) el intervalo diagnóstico fue 0 a $24 \mathrm{~h}$, en 67 pacientes (37\%) fue 25 a $48 \mathrm{~h}(\mathrm{p}=0,002), 38$ pacientes $(21 \%)$ se presentaron entre 49 y $72 \mathrm{~h}$ y 33 pacientes (18\%) llegaron después de $73 \mathrm{~h}$. El análisis de los criterios SIRS muestra que 73 pacientes $(40 \%)$ tenían una temperatura igual o mayor a $38^{\circ} \mathrm{C}$, en 119 casos $(65 \%)$ la frecuencia cardiaca fue $90 \times$ min o mayor, 27 pacientes $(15 \%)$ tuvieron una frecuencia respiratoria de $20 \mathrm{x}$ min o mayor y 152 pacientes $(83 \%, \mathrm{p}<0,0001)$ presentaron un RL mayor a $12.000 \mathrm{~mm}^{3}$. De acuerdo con la puntuación SIRS, 11 pacientes $(6 \%)$ tuvieron
0 puntos, 32 pacientes (17\%) tuvieron 1 punto, 83 pacientes $(45 \%, \mathrm{p}<0,0001)$ tuvieron 2 puntos, 42 pacientes $(23 \%)$ presentaron 3 puntos y 15 pacientes (8\%) tuvieron 4 puntos. Consecuentemente, 140 pacientes $(76,5 \%, \mathrm{p}<0,0001)$ presentaron SIRS.

\section{Características del SIRS}

En la Tabla 1 se observa la frecuencia de los criterios SIRS para cada grupo de intervalo diagnóstico. Un RL mayor a $12.000 \mathrm{~mm}^{3}$ fue más frecuente en los grupos 2 y 3 . Una temperatura mayor a $38^{\circ} \mathrm{C}$ fue más frecuente en el grupo 4. La frecuencia cardiaca mayor a $90 \mathrm{x}$ min se registró con frecuencia en el grupo 2, permaneciendo elevada en los grupos 3 y 4. La frecuencia respiratoria mayor a $20 \mathrm{x}$ min se presentó con frecuencia en el grupo 4. En la Tabla 2 se muestran los valores promedio de las variables del SIRS y la PCR, todos los promedios aumentaron progresivamente junto con el intervalo diagnóstico. La PCR llegó a su valor máximo en los grupos 3 y 4 ( $\mathrm{p}<0,0001)$. La Tabla 3 muestra la frecuencia de puntos SIRS, los pacientes del grupo 1 tuvieron menos puntos SIRS comparados con los otros grupos, además 49\% no tenían SIRS ( $\mathrm{p}<0,0001)$.

Tabla 1. Frecuencia de criterios SIRS en apendicitis de acuerdo a los grupos de intervalo diagnóstico

\begin{tabular}{|lcccc|}
\hline SIRS & $\begin{array}{c}\text { Grupo 1 } \\
\mathbf{( 0 - 2 4} \mathbf{~ h})\end{array}$ & $\begin{array}{c}\text { Grupo 2 } \\
\mathbf{( 2 5 - 4 8 ~ h )}\end{array}$ & $\begin{array}{c}\text { Grupo 3 } \\
(\mathbf{4 9 - 7 2} \text { h) }\end{array}$ & $\begin{array}{c}\text { Grupo 4 } \\
(>73 ~ \mathbf{~ h})\end{array}$ \\
Recuento de leucocitos $>12.000 \mathrm{~mm}^{3}$ & $35(78 \%)$ & $63(94 \%)^{*}$ & $35(92 \%)^{*}$ & $19(58 \%)$ \\
Temperatura $>38^{\circ} \mathrm{C}$ & $14(31 \%)$ & $20(30 \%)$ & $15(39,5 \%)$ & $24(73 \%)^{*}$ \\
Frecuencia cardíaca $>90 \mathrm{~min}$ & $12(27 \%)$ & $52(78 \%)^{*}$ & $29(76 \%)^{*}$ & $26(79 \%)^{*}$ \\
Frecuencia respiratoria $>20$ min & - & - & $3(8 \%)$ & $24(73 \%)^{*}$ \\
Total & $45(100 \%)$ & $67(100 \%)$ & $38(100 \%)$ & $33(100 \%)$ \\
\hline
\end{tabular}

$*_{p}<0,0001$.

Tabla 2. SIRS y PCR

\begin{tabular}{|lcccc|}
\hline Variable & $\begin{array}{c}\text { Grupo 1 } \\
\mathbf{( 0 - 2 4} \mathbf{~ h})\end{array}$ & $\begin{array}{c}\text { Grupo 2 } \\
\mathbf{( 2 5 - 4 8 ~ h )}\end{array}$ & $\begin{array}{c}\text { Grupo 3 } \\
\mathbf{( 4 9 - 7 2} \text { h) }\end{array}$ & $\begin{array}{c}\text { Grupo 4 } \\
(>73 \mathbf{~ h})\end{array}$ \\
Recuento de leucocitos $\left(\mathrm{mm}^{3}\right)$ & $16.020 \pm 3.784$ & $17.630 \pm 3.780$ & $18.037 \pm 5.759^{*}$ & $19.372 \pm 6.635^{*}$ \\
& $(8.300-22.700)$ & $(11.200-24.500)$ & $(6.200-26.700)$ & $(10.100-27.400)$ \\
Temperatura $\left({ }^{\circ} \mathrm{C}\right)$ & $37,7 \pm 0,4$ & $37,8 \pm 0,5$ & $37,8 \pm 0,9$ & $38,4 \pm 0,6^{* *}$ \\
& $(36,8-38,5)$ & $(36,5-38,9)$ & $(36-39,2)$ & $(37,5-39,4)$ \\
Frecuencia cardíaca $(\mathrm{min})$ & $91,3 \pm 14,1$ & $106,4 \pm 14,6 * *$ & $110 \pm 22,5^{* *}$ & $125,8 \pm 16^{* *}$ \\
& $(67-116)$ & $(80-124)$ & $(84-128)$ & $(92-131)$ \\
Frecuencia respiratoria $(\mathrm{min})$ & $16,4 \pm 1,4$ & $16,9 \pm 1,4$ & $18,3 \pm 1,3$ & $22,6 \pm 2,8 * *$ \\
& $(14-19)$ & $(14-19)$ & $(15-23)$ & $(18-28)$ \\
Proteína C reactiva $(\mathrm{mg} / \mathrm{dL})$ & $162,7 \pm 95$ & $210,5 \pm 142$ & $231 \pm 121 * *$ & $257,4 \pm 109 * *$ \\
& $(39,2-400,3)$ & $(63-360,5)$ & $(90,5-410,3)$ & $(95,8-450,3)$ \\
\hline
\end{tabular}

$* \mathrm{p}=0,003, * * \mathrm{p}>0,0001$ 
Tabla 3. Distribución de los puntos SIRS por grupo de intervalo diagnóstico

\begin{tabular}{|ccccc|}
\hline SIRS & $\begin{array}{c}\text { Grupo 1 } \\
(\mathbf{0 - 2 4} \text { h) }\end{array}$ & $\begin{array}{c}\text { Grupo 2 } \\
(\mathbf{2 5 - 4 8 ~ h )}\end{array}$ & $\begin{array}{c}\text { Grupo 3 } \\
(\mathbf{4 9 - 7 2} \mathbf{~ h})\end{array}$ & $\begin{array}{c}\text { Grupo 4 } \\
(>\mathbf{7 3} \mathbf{~ h})\end{array}$ \\
0 & $7(16 \%)^{*}$ & $4(6 \%)$ & - & - \\
1 & $15(33 \%)^{*}$ & $9(13 \%)$ & $5(13 \%)$ & $3(9 \%)$ \\
2 & $23(51 \%)^{*}$ & $44(66 \%)^{*}$ & $12(32 \%)$ & $4(12 \%)$ \\
3 & - & $10(15 \%)$ & $21(55 \%)^{*}$ & $11(33 \%)^{*}$ \\
4 & - & - & - & $15(45 \%)^{*}$ \\
Total & $45(100 \%)$ & $67(100 \%)$ & $38(100 \%)$ & $33(100 \%)$ \\
\hline
\end{tabular}

$* \mathrm{p}<0,0001$.

La mayoría de los pacientes del grupo 2 tuvieron 2 puntos SIRS $(66 \%, \mathrm{p}<0,0001) ; 13$ pacientes $(19 \%)$ no tenían SIRS y 10 pacientes $(15 \%)$ tuvieron 3 puntos SIRS. La mayoría de los pacientes del grupo 3 tenían SIRS (87\%), 21 pacientes $(55 \%)$ tuvieron 3 puntos SIRS ( $p<0,0001)$. Finalmente, $45 \%$ de los pacientes del grupo 4 tuvieron 4 puntos SIRS $(\mathrm{p}<$ 0,0001).

El análisis de las variables del SIRS de acuerdo a los intervalos diagnóstico, no mostró diferencias significativas en $\mathrm{RL}(\mathrm{p}=0,475)$ (Figura 1A). La temperatura se incrementa en forma constante después de $48 \mathrm{~h}$ alcanzando su nivel máximo después de $73 \mathrm{~h}(\mathrm{p}=0,001)$ (Figura 1B). La frecuencia cardiaca aumenta progresivamente desde el inicio de los síntomas presentando sus valores máximos después de $73 \mathrm{~h}(\mathrm{p}<0,0001)$. La frecuencia respiratoria aumenta después de $73 \mathrm{~h}(\mathrm{p}<0,0001)$ (Figura $1 C)$. Los valores de la PCR aumentan progresivamente junto con el intervalo diagnóstico mostrando niveles máximos después de $73 \mathrm{~h}(\mathrm{p}<0,0001)$. La frecuencia respiratoria aumenta después de $73 \mathrm{~h}(\mathrm{p}<$ 0,0001) (Figura 1C). Los valores de PCR aumentan progresivamente junto con la progresión del cuadro clínico, llegando a sus valores máximos después de $48 \mathrm{~h}(\mathrm{p}=0,005)$ (Figura 1D). Después de $72 \mathrm{~h}$ la mayoría de los pacientes tuvieron 3 ó 4 puntos SIRS $(\mathrm{p}<0,0001)$ (Figura 1E).

\section{Apendicitis perforada, complicaciones postopera- torias y correlaciones con intervalo diagnóstico y puntos SIRS}

La incidencia de apendicitis perforada de acuerdo con el intervalo diagnóstico se muestra en la Tabla 4 , debe notarse el aumento de la incidencia junto con la progresión del intervalo diagnóstico. La incidencia de perforación de acuerdo al SIRS se muestra en la Tabla 5, los pacientes con SIRS 4 tuvieron la mayor incidencia. Se evaluaron las correlaciones entre perforación, intervalo diagnóstico y SIRS mediante el test de Pearson, que demostró que la incidencia de perforación se relaciona a un intervalo diagnóstico mayor y a un puntaje SIRS mayor $(\mathrm{p}<0,0001)$. Se presentaron 7 complicaciones $(4 \%)$, en 6 casos fueron infecciones menores del sitio quirúrgico superficial y un caso de trombosis venosa profunda, este paciente tenía 3 puntos SIRS y un intervalo diagnóstico mayor a $73 \mathrm{~h}$ (Tabla 6).

Tabla 4. Incidencia de apendicitis perforada de acuerdo al intervalo diagnóstico

\begin{tabular}{|lc|}
\hline Intervalo diagnóstico & Tasa de perforación: n (\%) \\
Grupo 1: $0-24 \mathrm{~h}$ & $3(7)$ \\
Grupo 2: $25-48 \mathrm{~h}$ & $16(24)$ \\
Grupo 3: $49-72 \mathrm{~h}$ & $20(53)^{*}$ \\
Grupo 4: $>73 \mathrm{~h}$ & $28(85)^{*}$ \\
Total & $67(37)$ \\
\hline
\end{tabular}

$* \mathrm{p}<0,0001$.

Tabla 5. Apendicitis perforada de acuerdo al puntaje SIRS

\begin{tabular}{|cc|}
\hline SIRS & Tasa de perforación: n (\%) \\
0 & - \\
1 & $10(31)$ \\
2 & $17(20)$ \\
3 & $28(66)^{*}$ \\
4 & $12(80)^{*}$ \\
Total & $67(37)$ \\
\hline
\end{tabular}

$* \mathrm{p}<0,0001$.

Tabla 6. Complicaciones de acuerdo al intervalo diagnóstico y al puntaje SIRS

\begin{tabular}{|lc|}
\hline Intervalo diagnóstico & Complicaciones: $\mathbf{n}(\%)$ \\
Grupo 1: $0-24 \mathrm{~h}$ & $2(1)$ \\
Grupo 2: $25-48 \mathrm{~h}$ & $3(2)$ \\
Grupo 3: $49-72 \mathrm{~h}$ & - \\
Grupo 4: $>73 \mathrm{~h}$ & $2(1)$ \\
SIRS & \\
0 & - \\
1 & - \\
2 & $3(2)$ \\
3 & $3(2)$ \\
4 & $1(0,5)$ \\
\hline
\end{tabular}




\section{A. Recuento de leucocitos}

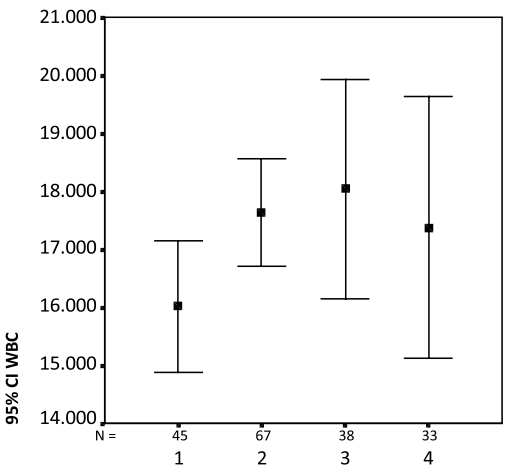

Diagnostic Interval

\section{B. Temperatura}

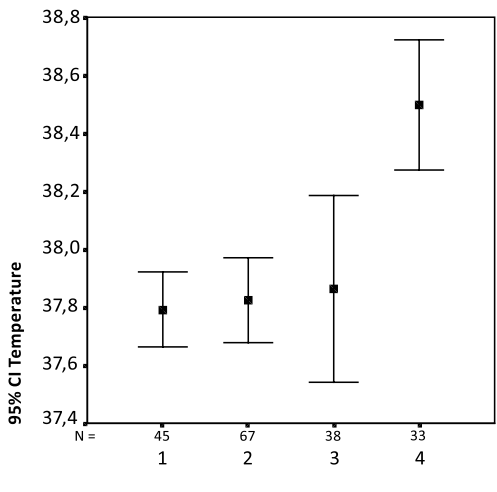

Diagnostic Interval

\section{Frecuencia respiratoria}

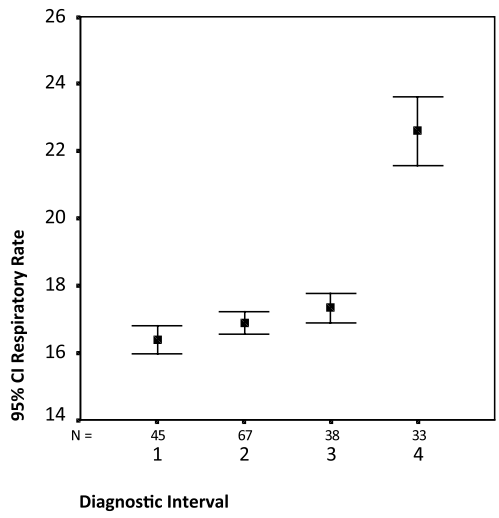

D. Proteína C reactiva

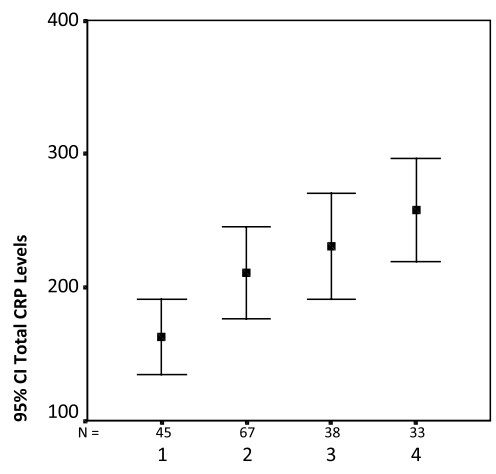

Diagnostic Interval
E. Puntos SIRS

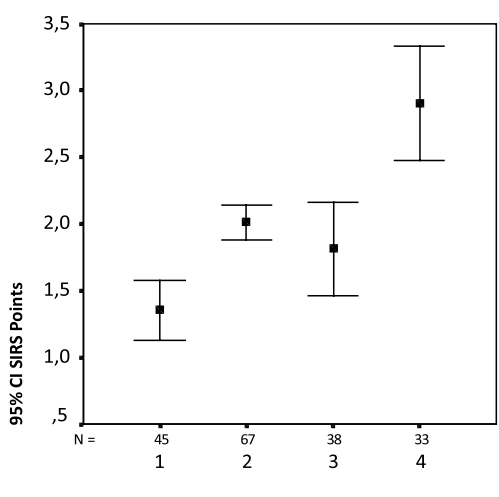

Diagnostic Interval

Figura 1. A) El recuento de los leucocitos aumentó con la progresión de la apendicitis. Después de 73 h desde el inicio de los síntomas, los valores promedio del recuento de leucocitos disminuyeron secundariamente a la adhesión de los leucocitos a las paredes capilares y posterior migración desde la circulación periférica hacia el área apendicular. B) La fiebre fue moderada durante las primeras $48 \mathrm{~h}$, después de este período la temperatura se eleva paulatinamente alcanzando sus máximos valores después de $73 \mathrm{~h}$. C) La frecuencia respiratoria permanece normal durante las primeras 72 $\mathrm{h}$, después de $73 \mathrm{~h}$ aumenta significativamente reflejando el compromiso séptico del paciente. D) La progresión de los valores de la proteína $\mathrm{C}$ reactiva fue constante y consecuente con la progresión del intervalo diagnóstico, siendo más marcada después de 48 h. E) El puntaje SIRS se incrementó con la progresión del intervalo diagnóstico, después de $73 \mathrm{~h}$ la mayoría de los pacientes tenía 3 ó 4 puntos SIRS. Esto es consecuente con la instauración del proceso séptico. 


\section{Discusión}

El diagnóstico y tratamiento precoz de la apendicitis disminuye las probabilidades del desarrollo de complicaciones sépticas, las que son más frecuentes en pacientes con apendicitis complicada ${ }^{2,5,13,14}$. Con un intervalo diagnóstico mayor a $72 \mathrm{~h}$ la presencia de apendicitis complicada es más frecuente y asociada a presentaciones atípicas que cuando el intervalo es menor a $12 \mathrm{~h}^{2,3,5,6,13-15}$, esta observación se confirma con el presente estudio que muestra intervalos diagnósticos y puntuaciones SIRS mayores relacionados a apendicitis perforada. La presentación tardía del paciente a los servicios de urgencia después del inicio de los síntomas se relaciona a una apendicitis más severa que el retraso intrahospitalario en la cirugía ${ }^{13}$. La información publicada demuestra la importancia de la reacción inflamatoria progresiva a apendicitis, la cual no ha sido propiamente descrita y caracterizada ${ }^{14}$. Este hecho es relevante debido a las recientes publicaciones sobre tratamiento conservador en apendicitis ${ }^{16-22}$, en estos casos la elevada tasa de fracaso del tratamiento y apendicitis recurrente revelan fallas en el diseño de estos estudios, los que utilizaron muestras no seleccionadas de pacientes con diferentes intervalos de diagnóstico sin tomar en cuenta la naturaleza evolutiva de esta patología.

La respuesta inflamatoria sistémica a apendicitis, no es uniformemente similar en todos los pacientes, en ese sentido se han reportado pacientes sin respuesta inflamatoria a apendicitis con frecuencias tan elevadas como $16 \%$ a $28 \%$, pero $52 \%$ a $80 \%$ de esos pacientes se presentaron temprano dentro del intervalo diagnóstico ${ }^{2,3}$. En el presente estudio, la frecuencia de pacientes sin respuesta inflamatoria sistémica fue $23,5 \%$, la mayor parte de ellos se presentaron tempranamente dentro del intervalo diagnóstico.

\section{Recuento de leucocitos}

La leucocitosis es el parámetro que con más frecuencia se espera encontrar en apendicitis y se considera como el mejor examen de laboratorio para el apoyo del diagnóstico clínico de apendicitis no complicada ${ }^{2,4,6,8,23,24}$, pero la ausencia de leucocitosis no descarta el diagnóstico de apendicitis $^{3,15,23,24}$. Se espera que pacientes con apendicitis avanzada tengan un RL elevado debido al aumento del recuento de polimorfonucleares ${ }^{5,14,24}$. Una vez que el proceso séptico se encuentra establecido se puede producir leucopenia. La leucocitosis se presentó en la mayoría de los pacientes de esta serie pero fue más frecuente en los grupos 2 y 3 . Esto es esperable debido a que la evolución clínica de la apendicitis llega a su punto máximo entre las
24 y $48 \mathrm{~h}$ desde el inicio de los síntomas $3,5,6,13,15$. La mayoría de los pacientes en el grupo 1 (78\%), tenían leucocitosis lo cual es esperable debido a la fase inicial del proceso inflamatorio ${ }^{3-6,24}$. En el grupo 4, 58\% de los pacientes tuvieron un RL elevado, algunos pacientes tuvieron RL normales, esta respuesta también es esperable debido a que la respuesta inflamatoria seguida de la instauración de sepsis después de $72 \mathrm{~h}$ eventualmente lleva a la disminución del RL o a leucopenia ${ }^{2,4,5,13,14,24-29}$. En estudios que utilizaron diferentes intervalos diagnósticos la incidencia de leucocitosis varió entre $78 \%$ y $88 \% \%^{2,3}$. En otro estudio que utilizó 3 diferentes intervalos diagnósticos la presencia de leucocitosis aumentó junto con el período de evolución de los síntomas ${ }^{14}$. Un elevado RL puede esperarse en pacientes con apendicitis avanzada ${ }^{5,6,14,24}$; sin embargo, la mayoría de los pacientes que se presentan tardíamente pueden tener un RL disminuido comparado con pacientes que se presentan precozmente ${ }^{4-6,15}$, lo cual es similar a los resultados del presente estudio. Consecuentemente, debe esperarse que el RL aumente progresivamente junto con el intervalo diagnóstico aunque después de $73 \mathrm{~h}$ el $\mathrm{RL}$ disminuiría manteniendo un recuento elevado en aproximadamente $58 \%$ de los casos.

\section{Temperatura}

La temperatura en la apendicitis es moderada aun en casos complicados ${ }^{2}$. En pacientes con apendicitis inicial o avanzada la ausencia de fiebre se ha reportado con una frecuencia de $28 \%$ y $14 \%$ respectivamente ${ }^{3}$. Fiebre elevada sobre $38^{\circ} \mathrm{C}$, se ha reportado en $56 \%$ de los pacientes con apendicitis inicial y en $74 \%$ con apendicitis avanzada ${ }^{2,3}$. Habitualmente, cuando se presenta fiebre elevada se debe a peritonitis o absceso y representa una apendicitis avanzada con un prolongado intervalo diagnóstico ${ }^{3,24}$. La frecuencia de fiebre mayor a $38^{\circ} \mathrm{C}$ fue de $30 \%$ a $40 \%$ para los grupos 1 y 3 , en el grupo 4 el $73 \%$ de los pacientes tuvieron una temperatura mayor a $38^{\circ} \mathrm{C}$. La temperatura aumentó progresivamente durante las primeras $72 \mathrm{~h}$ desde el inicio de los síntomas con un valor promedio de $37,8^{\circ} \mathrm{C}$, después de 73 h la temperatura aumentó hasta llegar a un valor promedio de $38,4^{\circ} \mathrm{C}$.

\section{Frecuencia cardíaca}

El aumento de la frecuencia cardíaca es esperable de acuerdo al aumento de la temperatura, ambas respuestas mediadas por la producción local y sistémica de mediadores inflamatorios como interleucinas y fosofolipasas ${ }^{2,4}$. La frecuencia cardíaca alcanza valores elevados rápidamente después de $24 \mathrm{~h}$ desde el inicio de los síntomas permaneciendo elevada en todos los intervalos de diagnóstico posteriores. 


\section{Frecuencia respiratoria}

La frecuencia respiratoria se presentó elevada en $15 \%$ de todos los pacientes de la serie, principalmente en el grupo de pacientes con más de $73 \mathrm{~h}$ de intervalo diagnóstico (73\% de los casos). Una frecuencia de $34 \%$ se ha reportado para este parámetro del SIRS en una cohorte de pacientes con diferentes intervalos diagnóstico ${ }^{2}$. El aumento de la frecuencia respiratoria después de $72 \mathrm{~h}$ denota un estado séptico severo y acidosis metabólica.

\section{SIRS}

La frecuencia de SIRS en pacientes con apendicitis se ha reportado entre $50 \%$ y $86 \%^{2,5}$. En el presente estudio fue de $76,5 \%$. La duración de los síntomas desde el inicio hasta el diagnóstico se relaciona con la severidad de la apendicitis ${ }^{2,5}$, en este sentido era esperable que la duración de los intervalos diagnósticos se correlacionara con respuestas inflamatorias más severas, lo cual se confirma por los hallazgos de esta serie. Pacientes con menos de $24 \mathrm{~h}$ de intervalo diagnóstico tuvieron entre 0 y 2 puntos SIRS. Cuando el intervalo diagnóstico fue de 25 a $48 \mathrm{~h}$, todos los pacientes tuvieron entre 1 y 3 puntos SIRS, la mayoría de ellos tuvieron 2 puntos y los parámetros elevados con mayor frecuencia fueron el RL y la frecuencia cardíaca. Pacientes con un intervalo diagnóstico de 49 a 72 h tuvieron entre 1 y 3 parámetros SIRS, la mayoría de ellos tuvieron 3 puntos SIRS los más frecuentes de los cuales fueron el aumento del RL y de la frecuencia cardíaca. Finalmente, pacientes con un intervalo diagnóstico mayor a $73 \mathrm{~h}$ tuvieron 3 ó 4 puntos SIRS siendo los más frecuentemente elevados la temperatura, la frecuencia cardíaca y la frecuencia respiratoria. El RL aumentó constantemente durante el intervalo diagnóstico de 0 a $72 \mathrm{~h}$, después de 73 h disminuyó. Esto se explica porque después de 73 $h$ los pacientes se encuentran en un estado séptico y los leucocitos se han marginalizado y migran hacia el área inflamada y consecuentemente, sus números disminuyen. Otros parámetros del SIRS se elevan debido a que en casos de apendicitis avanzada la fosfolipasa $\mathrm{A}_{2}$ y las interleucinas IL1 $\beta$, IL6, IL8, e IL10 aumentan localmente y sistémicamente. Además, el desarrollo de acidosis metabólica aumenta la frecuencia respiratoria ${ }^{5}$.

\section{Proteína C reactiva}

La PCR es un marcador inflamatorio inespecífico útil en el diagnóstico de apendicitis junto con el RL y los parámetros clínicos ${ }^{3,5,6,8-10}$. Los valores elevados de la PCR se asocian a apendicitis, esta asociación es más fuerte cuanto mayor es el intervalo diagnóstico y mayores son los valores de la $\mathrm{PCR}^{3-6,8,24}$. Se ha demostrado que valores elevados de PCR se asocian a apendicitis perforada ${ }^{5,6,24}$. Consecuentemente, la PCR se considera como un mejor marcador serológico para apendicitis avanzada que el RL ${ }^{4-6,24}$. En este estudio, los valores de la PCR aumentaron constantemente dentro del intervalo diagnóstico alcanzando sus valores máximos después de $48 \mathrm{~h}$, como ha sido previamente descrito ${ }^{5,6,24}$.

\section{Conclusiones}

La respuesta inflamatoria a la apendicitis es progresiva, siendo más severa a lo largo de la línea de tiempo desde el inicio de los síntomas hasta el diagnóstico, correlacionándose con una presentación avanzada.

\section{Referencias}

1. Stephenson JA, Gravante G, Butler NA, Sorge R, Sayers RD, Bown MJ. The systemic inflammatory response (SIRS) - Number and type of positive criteria predict interventions and outcomes in acute surgical admissions. World J Surg. 2010;34:2757-64.

2. Rivera-Chavez FA, Wheeler H, Lindberg G, Munford RS, O'Keefe GE. Regional and systemic cytokine responses to acute inflammation of the vermiform appendix. Ann Surg. 2003;237:408-16.

3. Andersson RE, Hugander AP, Ghazi SH, Ravn H, Offenbartl SK, Nyström PO, et al. Diagnostic value of disease history, clinical presentation, and inflammatory parameters of appendicitis. World J Surg. 1999;23:13348.

4. Grönroos JM, Forsström JJ, Irjala K, Nevalainen TJ. Phospholipase A2, C-reactive protein and white blood cell count in the diagnosis of acute appendicitis. Clin Chem. 1994;40:1757-60.

5. Beltrán MA, Barrera RC, Méndez PP, Contreras MG, Wilson CG, Cortés VJ, y cols. Hiperbilirubinemia en apendicitis - ¿Es un factor predictivo de perforación? Rev Chil Cir. 2009;61:413-22.

6. Ortega-Deballon P, Ruiz de Adana-Belbel JC, Hernández-Matías $\mathrm{A}$, García-Septiem J, Moreno-Azcoita M. Usefulness of laboratory data in the management of right iliac fossa pain in adults. Dis Colon Rectum 2008;51:1093-9.

7. Andersson M, Andersson RE. The appendicitis inflammatory response score: A tool for the diagnosis of acute appendicitis that outperforms the Alvarado score. World J Surg. 2008;32:1843-9.

8. De Castro SM, Ünlü Ç, Steller EPh, van Wagensveld BA, Vrouenraets BC. Evaluation of the appendicitis inflammatory response score for patients with acute appendicitis. World J Surg. 2012;36:1540-5.

9. Beltrán MA, Villar RM, Tapia TF. Score diagnóstico 
de apendicitis: Estudio prospectivo, doble ciego, no aleatorio. Rev Chil Cir. 2004;56:550-7.

10. Beltrán MA, Villar R, Cruces KS. Puntuación diagnóstica para apendicitis: Estudio prospectivo de su aplicación por profesionales de salud no-médicos. Rev Med Chile 2006;134:39-47.

11. Levy MM, Fink MP, Marshall JC, Abraham E, Angus D, Cook D, et al; International Sepsis Definitions Conference. $2001 \mathrm{SCCM} / \mathrm{ESICM} / \mathrm{ACCP} / \mathrm{ATS} / \mathrm{SIS}$ International Sepsis Definitions Conference. Intensive Care Med. 2003;29:530-8.

12. Beltrán MA. Hemicolectomía derecha en pacientes operados por apendicitis aguda: De la incisión de McBurney y apendicectomía a la laparostomía mediana y hemicolectomía. Rev Colomb Cir. 2012;27:129-38.

13. Ditillo MF, Dziura JD, Rabinovici R. Is it safe to delay appendectomy in adults with acute appendicitis? Ann Surg. 2006;244:656-60.

14. Wu HP, Huang CY, Chang YJ, Chou CC, Lin CY. Use of changes over time in serum inflammatory parameters in patients with equivocal appendicitis. Surgery 2006;139:789-96.

15. Beltrán MA, Villar RM, Tapia TF, Cruces KS. Sintomatología atípica en 140 pacientes con apendicitis. Rev Chil Cir. 2004;56:269-74.

16. Hansson J, Körner U, Ludwigs K, Johnsson E, Jönsson C, Lundholm K. Antibiotics as first-line therapy for acute appendicitis: Evidence for a change in clinical practice. World J Surg. 2012;36:2028-36.

17. Fitzmaurice GJ, McWilliams B, Hurreiz H, Epanomeritakis E. Antibiotics versus appendectomy in the management of acute appendicitis: A review of the current evidence. Can J Surg. 2011;54:307-14.

18. Mason RJ. Surgery for appendicitis: Is it necessary? Surg Infect 2008;9:481-8.

19. Andersson RE, Petzold MG. Nonsurgical treatment of appendiceal abscess or phlegmon: A systematic review and metanalysis. Ann Surg. 2007;246:741-8.

20. Styrud J, Eriksson S, Nilsson I. Ahlberg G, Haapaniemi
S, Neovius G, et al. Appendectomy versus antibiotic treatment in acute appendicitis. a prospective multicenter randomized controlled trial. World J Surg. 2006;30:1033-7.

21. Hansson J, Körner U, Khorram-Manesh A, Solberg A, Lundholm K. Randomized clinical trial of antibiotic therapy versus appendicectomy as primary treatment of acute appendicitis in unselected patients. Br J Surg. 2009;96:473-81.

22. Malik AA, Bari U. Conservative management of acute appendicitis. J Gastrointest Surg 2009;13:966-70.

23. Monneuse O, Abdalla S, Pilleul F, Hervieu V, Gruner L, Tissot E, Barth X. Pain as the only consistent sign of acute appendicitis: Lack of inflammatory signs does not exclude the diagnosis. World J Surg. 2010;34:210-5.

24. Andersson RE, Hugander A, Ravn H, Offenbartl K, Ghazi SH, Nyström PO, Olaison G. Repeated clinical and laboratory examinations in patients with an equivocal diagnosis of appendicitis. World J Surg. 2000;24:479-85.

25. Guidet B, Aegerter P, Gauzit R, Meshaka P, Dreyfuss D. Incidence and impact of organ dysfunctions associated with sepsis. Chest 2005; 127:942-51.

26. Schein M, Marshall J. Source control for surgical infections. World J Surg. 2004;28:638-45.

27. De Waele JJ. Early source control in sepsis. Langenbecks Arch Surg. 2010;395:489-94.

28. Hynninen M, Wennervirta A, Leppäniemi A, Pettilä V. Organ dysfunction and long term outcome in secondary peritonitis. Langenbecks Arch Surg. 2008;393:81-6.

29. Zhao H, Heard SO, Mullen MT, Crawford S, Goldberg RJ, Frendl G, et al. An evaluation of the diagnostic accuracy of the 1991 American College of Chest Physicians/Society of Critical Care Medicine and the 2001 Society of Critical Care Medicine/European Society of Intensive Care Medicine/American College of Chest Physicians/American Thoracic Society/Surgical Infection Society sepsis definition. Crit Care Med. 2012;40:1700-6. 